\title{
T-SCAN: OBTENCIÓN DE NUBES DE PUNTOS CON COLOR Y TEMPERATURA EN INTERIOR DE EDIFICIOS
}

\author{
T. Prado, B. Quintana, S. A. Prieto, A. Adán \\ 3D Visual Computing and Robotics Lab, Universidad de Castilla-La Mancha. Ciudad Real, España. \\ Tomas.Prado@alu.uclm.es, \{Blanca.Quintana, Samuel.Prieto, Antonio.Adan\}@uclm.es
}

\begin{abstract}
Resumen
Este articulo presenta el sistema T-Scan (Thermal Scan) dedicado a realizar una captación de nubes de puntos densas con información de color $y$ temperatura. El sistema forma parte del proyecto $T$ BIM (Thermal Building Information Models) en el que se pretende generar de forma automática modelos semánticos térmicos de edificios, con el objetivo de integrarlos en los actuales modelos normalizados BIM. El artículo presenta el sistema sensorial, la calibración, metodología y los resultados experimentales en entornos reales.
\end{abstract}

Palabras Clave: Tecnologías láser escáner, Imagen térmica, Procesamiento de datos 3D, modelado 3D, BIM.

\section{INTRODUCCIÓN}

El proceso de generación de modelos 3D a partir de datos de nubes de puntos para ser usados con fines de modelado de energía consiste básicamente en tres etapas: colección de datos, modelado y análisis. En la práctica, la toma de datos se lleva a cabo de forma manual por auditores de energía, tomando muestras locales de la escena. La ejecución manual de estas tareas puede ser lenta, costosa e incompleta.

El desarrollo de métodos automáticos de toma de datos para generar modelos 3D térmicos densos de interiores de edificios es una línea puntera que entrará en breve a formar parte del proceso global de creación de modelos BIM. Este artículo está dedicado a exponer el componente sensorial y la creación de una de nube de puntos térmica que es utilizada en las etapas posteriores de modelado semántico y análisis de la información 3D térmica.

\section{ESTADO DEL ARTE}

La adquisición y modelado de datos $3 \mathrm{D}$ es un campo de investigación tratado desde hace varios años por multitud de autores. Sin embargo, la inclusión de información térmica en datos $3 \mathrm{D}$ es un aspecto novedoso y que comienza a ser de gran interés en la comunidad científica [2].

La idea principal consiste en incorporar una cámara térmica a un sistema de adquisición de datos 3D para aportar información térmica a una nube de puntos. Se pueden encontrar distintos sistemas, desde sistemas con fotogrametría [4] y cámaras de profundidad [9] hasta sistemas más precisos basados en escáneres 3D y tecnología LiDAR $[1,3,6-8,10]$.

La operación clave del proceso consiste en calibrar ambos sistemas de adquisición entre sí, de manera que los datos térmicos puedan proyectarse sobre la nube de puntos 3D. Ham et al. [4] presentan un sistema únicamente compuesto por una cámara térmica FLIR E60, capaz de adquirir tanto imágenes térmicas como digitales. Un modelo 3D, tanto térmico como espacial, es generado mediante una calibración extrínseca e intrínseca (realizada con un panel compuesto por 42 bombillas LED). Posteriormente los modelos son alineados tomando puntos característicos elegidos por un usuario. Rangel et al. [9] usan un sistema compuesto por una cámara de profundidad Microsoft Kinect y una cámara térmica Jenoptik IR-TCM 640. Se sirve de un panel de calibración para obtener la relación geométrica entre ambas cámaras. Realizan un estudio exhaustivo sobre el material utilizado en dicho panel y la distribución geométrica del mismo, ya que los puntos de referencia deben ser visibles tanto en la imagen de profundidad como en la térmica, siendo finalmente utilizado un panel de cartón pluma con orificios circulares equi-espaciados. Borrmann et al. [1] presentan un sistema compuesto por un láser escáner 3D Riegl VZ-400 y una cámara térmica Optris PI160. Un panel de calibración es de nuevo usado para obtener la relación entre ambos sistemas de referencia, estando en este caso compuesto por 30 bombillas incandescentes. El sistema de adquisición de Mader et al. [8] difiere de los anteriores en el sentido de que no es un sistema integrado en un solo dispositivo, sino que se compone de tres drones cada uno equipado con un sensor diferente. Uno de los drones, equipado con un láser de rango Hokuyo UTM-30LX se encarga de obtener una nube de puntos 3D del escenario. Mientras que los otros dos drones, equipados con una cámara RGB y una cámara térmica FLIR A65 
respectivamente, obtienen los datos de color e información térmica. La calibración entre los datos geométricos y las imágenes se realiza mediante un patrón compuesto por marcadores identificables por todos los sensores distribuido en el suelo. De manera similar a los métodos expuestos anteriormente, Chao Wang et al. [10] utilizan un patrón con forma de tablero de ajedrez para registrar los datos obtenidos mediante un LiDAR y una cámara térmica. Dicho patrón contiene huecos en las casillas blancas por los que, al ser colocado frente a un cuerpo caliente, se puede visualizar tanto en la imagen térmica como en la nube de puntos características comunes para realizar el registro.

Todos estos artículos presentan sistemas integrados de adquisición de información 3D con datos de temperatura. Sin embargo, otros autores proponen metodologías que permiten combinar información $3 \mathrm{D}$ con información térmica adquirida con sistemas independientes. Lagüela et al. [6] proponen una metodología que permite obtener la relación entre los datos geométricos y térmicos mediante un software comercial, eligiendo de manera manual puntos en común entre ambos escenarios y registrando así los datos. Para la adquisición de datos utilizan un láser escáner 3D Riegl LMS-Z390i y una cámara térmica NEC TH9260. En este caso el panel de calibración (compuesto por 64 bombillas) es utilizado para la calibración intrínseca de la cámara térmica y posterior corrección de la distorsión. Por el contrario, GonzálezAguilera et al. [3] presentan una metodología para el registro automático de la información de ambos sensores, identificando puntos singulares tanto en la nube de puntos 3D (obtenida mediante un Faro Photon 80) como en las imágenes térmicas (obtenidas mediante una FLIR ThermaCAM SC640), obteniendo así la transformación necesaria para proyectar la información térmica sobre la nube de puntos. Por último, López-Fernández [7] et al. también presentan un método que permite registrar la información de dos sistemas independientes. Por un lado, adquieren imágenes térmicas de cada pared con una cámara térmica NEC TH9260, y por otro adquieren nubes de puntos con un sistema de mapeado de interiores formado por un láser escáner 2D Hokuyo UTM-30LX, una IMU y dos encoders de canal dual. El proceso de registro entre ambos sistemas se realiza seleccionando características homólogas en ambas imágenes de manera manual.

\section{CONTRIBUCIONES DEL MÉTODO}

La diferencia fundamental del sistema que presenta este artículo radica en tres aspectos. En primer lugar, el sistema de calibrado es novedoso ya que se utilizan dianas (balizas) que incorporan a la vez discriminantes térmico y de reflectancia, lo que hace aumentar la precisión y eficiencia del sistema (Sección 5.2).
En segundo lugar, la posición de las dianas no está restringida a pequeñas regiones como en $[1,9,10]$ (contenidas en pequeños tableros) - frecuentemente balizas activas (bombillas) [1,8], identificadas en tableros $[9,10]$ o en características de la imagen $[3,4,6,7]$. Por el contrario, nuestras balizas pueden abarcan un espacio amplio de la escena, en posiciones lejanas del escáner, y donde no existen restricciones en su posicionamiento. Como consecuencia, la calibración es más fiable y precisa.

En tercer lugar, muchos de los sistemas referenciados no tratan el problema de completitud del espacio observable, por lo que solamente obtienen un mapa térmico parcial de la escena. El sistema T-Scan está preparado para realizar un mapa térmico 3D completo del espacio debido a que realiza una integración de tomas desde una o distintas posiciones, consiguiendo un mapa acumulado muestreado que cubre todo el escenario (Sección 6).

\section{3D T-SCAN}

El sistema escáner 3D térmico desarrollado se muestra en la Figura 1. Este sistema capta nubes de puntos con información de color (RGB) y temperatura. Consta del escáner 3D RIEGL VZ-400, la cámara RGB Nikon D90 y la cámara térmica FLIR Series AX5 A65.

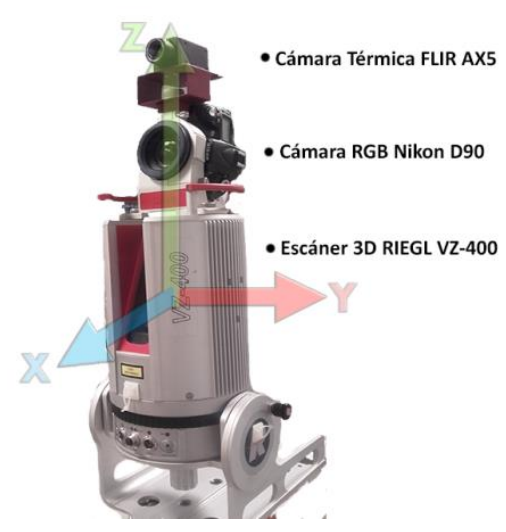

Figura 1: Imagen del sistema 3D T-Scan. Se ilustra el sistema de coordenadas universal utilizado.

El escáner 3D RIEGL VZ-400 es un escáner 3D láser terrestre capaz de captar coordenadas 3D de su entorno utilizando un haz láser de clase uno. Presenta una distancia máxima de escaneo de $600 \mathrm{~m}$, con un ángulo de visión horizontal de $360^{\circ}$ y vertical de $30^{\circ}$ a $130^{\circ}$. Posee un engranaje que le permite ser inclinado hasta $90^{\circ}$, permitiendo así escanear suelos y techos. Los rangos de paso de barrido horizontal y vertical son $\left[0.0024^{\circ}, 0.5^{\circ}\right]$ y $\left[0.0024^{\circ}, 0.28^{\circ}\right]$, permitiendo amoldarse a las necesidades del usuario. 
La cámara RGB Nikon D90 tiene una óptica con apertura $170^{\circ}$ y resolución de $4288 \times 2848$ píxeles. Por su parte la cámara térmica FLIR A65 posee una resolución de $640 \times 512$ píxeles y un campo de visión de $45 \times 37^{\circ}$ captados a una frecuencia de $30 \mathrm{~Hz}$. Se trata de una cámara muy versátil ya que permite ajustar la precisión y el rango de temperaturas captado gracias a la posibilidad de configurarla de dos modos, "HighMode", lo que nos dará un rango de temperaturas de $233-823^{\circ} \mathrm{K}$ con una precisión de $0.4^{\circ}$ y "LowMode", que permite un rango de temperaturas de $248-408^{\circ} \mathrm{K}$ con una precisión de $0.04^{\circ}$.

El sistema se ha diseñado para funcionar en dos fases (ver Figura 2). La fase 1 consiste en la calibración de los elementos del sistema y es necesaria ejecutarla una única vez. Los resultados de calibración son usados como base para la toma de datos 3D-térmicos en la fase 2.

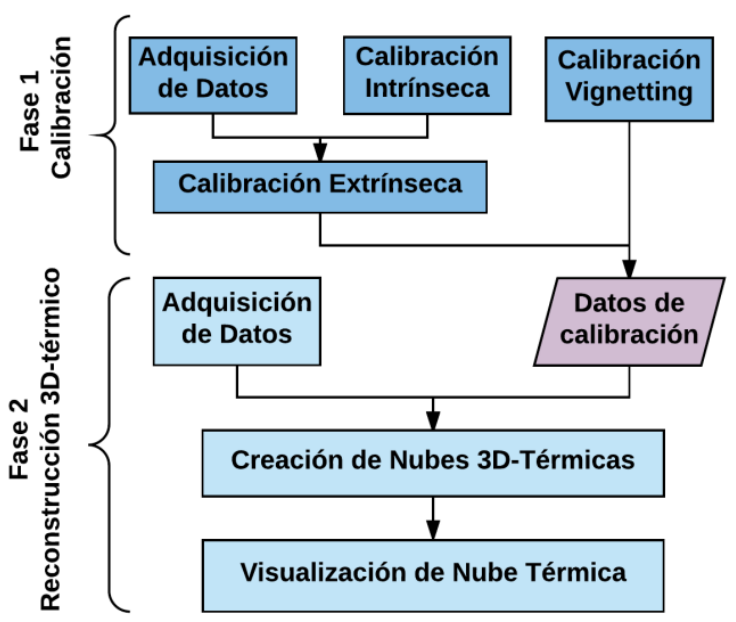

Figura 2: Esquema funcionamiento del escáner 3D térmico

\section{CALIBRACIÓN DEL SISTEMA}

En primer lugar, es necesario llevar a cabo un conjunto de calibraciones, no sólo de cada elemento del sistema por separado, sino también de la integración entre los mismos.

\subsection{CALIBRACIÓN DE LAS CÁMARAS}

Con objeto de obtener imágenes de máxima calidad se realiza un proceso de calibración intrínseco en donde se obtienen los parámetros que regulan la distorsión presente en la imagen, tanto para la cámara RGB como para la cámara térmica. El procedimiento usado para obtener los parámetros intrínsecos ha sido el seguido por Heikkilä et al. [5]. Con los parámetros intrínsecos de distorsión radial $(k 1, k 2, k 3)$ y tangencial $(p 1, p 2)$, se calcula la nueva posición de los píxeles $\left(X_{n}, Y_{n}\right)$ de la imagen sin distorsión como:
$X n=X\left(1+k_{1} r^{2}+k_{2} r^{4}+k_{3} r^{6}\right)+2 Y p_{1}+p_{2}\left(r^{2}+2 X^{2}\right)$

$Y n=Y\left(1+k_{1} r^{2}+k_{2} r^{4}+k_{3} r^{6}\right)+2 X p_{2}+p_{1}\left(r^{2}+2 Y^{2}\right)$

Con $r=\sqrt{X^{2}+Y^{2}}$

La cámara térmica además presenta una distorsión conocida como vignetting, la cual produce un ennegrecimiento de los bordes de la imagen, falseando de esta manera la información térmica obtenida (ver Figura 3). Para lograr corregir esta distorsión es necesario conocer el porcentaje de pérdida de temperatura en función de su distancia con el centro de la imagen.

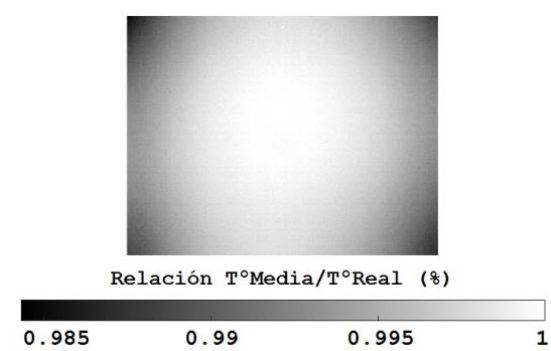

Figura 3: Efecto vignetting observado en una imagen térmica

La calibración del efecto vignetting ha sido modelada utilizando un panel de madera de temperatura conocida y constante. La calibración proporciona una función de pérdida radial de temperatura. Para obtener los valores corregidos en la imagen térmica sólo es necesario dividir cada píxel de la imagen entre su porcentaje de pérdida. Los resultados obtenidos se muestran en la Figura 4.

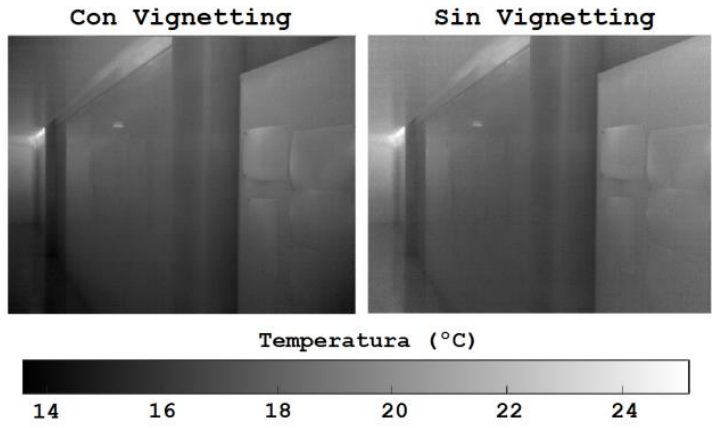

Figura 4: Imagen térmica corregida de vignetting

\subsection{CALIBRACIÓN DEL SISTEMA ESCÁNER- CÁMARA TÉRMICA}

La asociación entre píxeles de la imagen térmica $\boldsymbol{I}_{T}$ con puntos de la nube 3D captada por el escáner requiere calcular la matriz de transformación proyectiva correspondiente. Esta es una transformación que relaciona las coordenadas de la nube de puntos con las coordenadas, en píxeles, de los 
correspondientes puntos proyectados en la imagen térmica. La transformación se modela en la ecuación siguiente:

$$
\left(\begin{array}{c}
\lambda X_{f} \\
\lambda Y_{f} \\
\lambda
\end{array}\right)=\left(\begin{array}{llll}
r_{11} & r_{12} & r_{13} & r_{14} \\
r_{21} & r_{22} & r_{23} & r_{24} \\
r_{31} & r_{32} & r_{33} & r_{34}
\end{array}\right)\left(\begin{array}{c}
X_{p} \\
Y_{p} \\
Z_{p} \\
1
\end{array}\right)
$$

la ecuación (2) puede ser expresada como:

$$
\left(\begin{array}{l}
X_{f} \\
Y_{f}
\end{array}\right)=\left(\begin{array}{lllllllllll}
X_{p} & Y_{p} & Z_{p} & 1 & 0 & 0 & 0 & 0 & -X_{f} X_{p} & -X_{f} Y_{p} & -X_{f} Z_{p} \\
0 & 0 & 0 & 0 & X_{p} & Y_{p} & Z_{p} & 1 & -Y_{f} X_{p} & -Y_{f} Y_{p} & -Y_{f} Z_{p}
\end{array}\right)\left(\begin{array}{l}
r_{11} \\
r_{12} \\
r_{13} \\
r_{14} \\
r_{21} \\
r_{22} \\
r_{24} \\
r_{31} \\
r_{32} \\
r_{33}
\end{array}\right)
$$

Para $n$ pares de coordenadas correspondientes, se forma un sistema sobredeterminado:

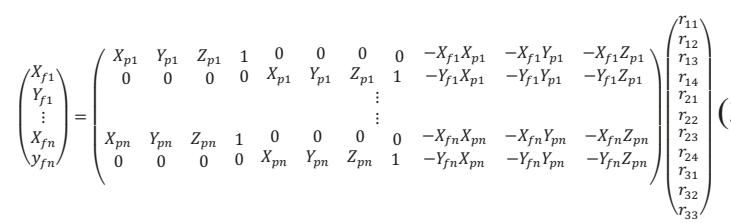

que puede ser expresado como $\boldsymbol{C}=\boldsymbol{W} \boldsymbol{P}$.

Conocidas las coordenadas de los píxeles $\left(X_{f}, Y_{f}\right)$, las correspondientes coordenadas $3 \mathrm{D}$ de los puntos asociados $\left(X_{p}, Y_{p}, Z_{p}\right)$, e imponiendo $r_{34}=1$, la ecuación (3) queda resuelta por:

$$
P=\left(W^{T} W\right)^{-1} W^{T} C
$$

En la práctica, en el procedimiento de calibración se utilizan dianas reflectantes pegadas en pequeños cubos de plástico de hielo. Como se puede apreciar en la Figura 5, esto facilita la localización de los mismos tanto en la imagen de reflectancia asociada a la nube de puntos 3D como en la imagen térmica. El emparejamiento de puntos correspondientes se realiza mediante un algoritmo de búsqueda por posicionamiento de dianas en la imagen y posterior refinamiento verificando la coherencia entre los conjuntos correspondientes.

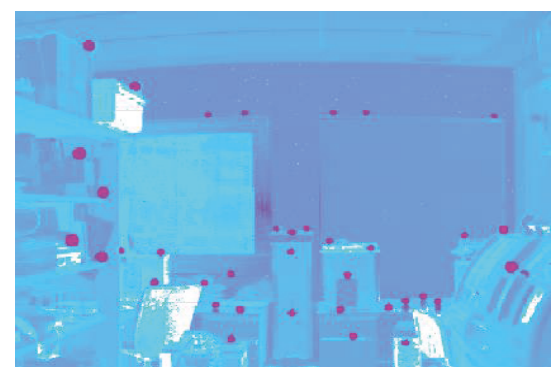

a)

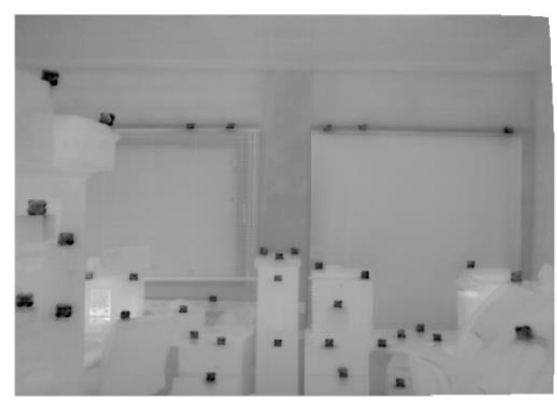

b)

Figura 5: Localización de dianas. a) Imagen de reflectancia. b) Imagen térmica

La matriz de transformación es recalculada minimizando el error entre las coordenadas obtenidas mediante la ecuación (2), $\left(X_{f}^{\prime}, Y_{f}^{\prime}\right)$, y las detectadas en la imagen, $\left(X_{f}, Y_{f}\right)$. Para ello se toma un conjunto de puntos vecinos del centroide de la diana, se aplica de nuevo la ecuación (4) y se toma la matriz de transformación que proporciona el mínimo error (ver diagrama de flujo de la Figura 6).

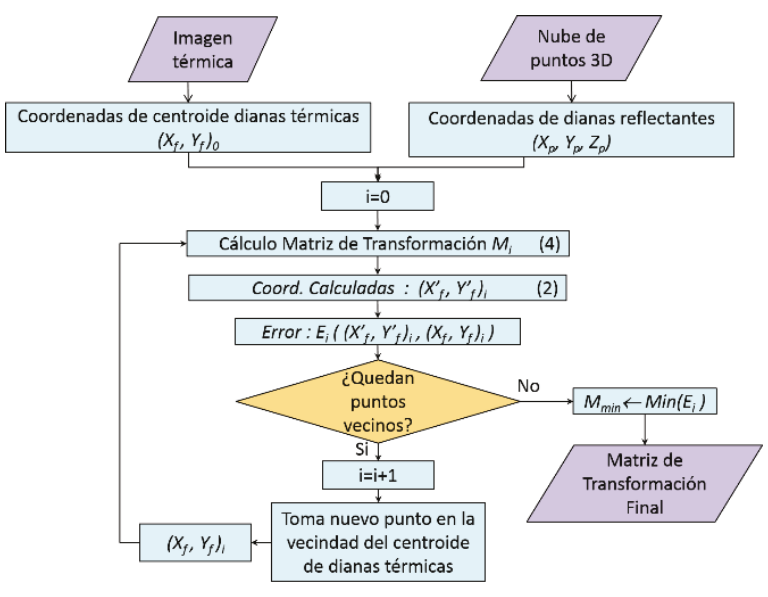

Figura 6: Refinamiento de la matriz de transformación proyectiva

\section{OBTENCIÓN DE LA NUBE DE PUNTOS TÉRMICA DEL ESPACIO OBSERVABLE}

\subsection{INTEGRACIÓN DE IMÁGENES TÉRMICAS EN LA NUBE DE PUNTOS}

El procedimiento seguido para la inclusión de un conjunto de imágenes térmicas en la nube de puntos está explicado en el diagrama de flujo de la Figura 7. 


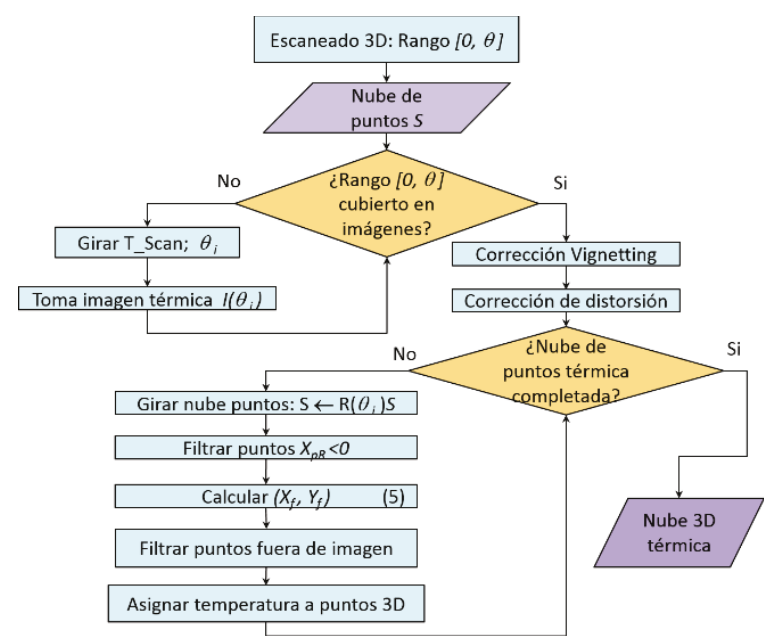

Figura 7: Proceso creación Nubes 3D térmicas

El sistema 3D T-Scan realiza la toma de datos en tres fases secuenciales. En la primera fase el escáner 3D capta las coordenadas 3D de puntos alcanzados por el láser en un espacio definido por el rango de ángulos vertical $\lambda$ y horizontal $\theta$ fijados por el usuario. Normalmente el rango de $\lambda$ se mantiene en $\left[30^{\circ}, 130^{\circ}\right]$, mientras que el rango de $\theta$ es fijado a voluntad.

En la segunda fase, las cámaras RGB y térmica realizan, partiendo de $\theta=0$, el número de tomas necesarias para cubrir totalmente el rango de $\theta$ elegido en la sesión. De acuerdo con el campo de visión de las cámaras, cada toma de imágenes se realiza después de girar el 3D T-Scan en $\theta$ lo necesario para que exista un mínimo solape entre imágenes térmicas consecutivas.

En la tercera fase se trata de preprocesar las imágenes y de asignar la temperatura a la nube de puntos tomada en la fase primera. El preprocesamiento consta esencialmente de la eliminación del vignetting y de la corrección de distorsión.

Realizadas $m$ tomas en los ángulos $\theta_{1}, \theta_{2}, \ldots \theta_{m}$, las coordenadas de los puntos proyectados en cada imagen térmica $I\left(\theta_{1}\right), I\left(\theta_{2}\right), \ldots I\left(\theta_{m}\right)$ son calculados después de haber incluido las correspondientes matrices de rotación $R\left(\theta_{1}\right), R\left(\theta_{2}\right), \ldots R\left(\theta_{m}\right)$ en la ecuación (2). Formalmente la expresión es:

$$
\left(\begin{array}{l}
X_{f} \\
Y_{f} \\
1
\end{array}\right)=\left(\begin{array}{llll}
r_{11} & r_{12} & r_{13} & r_{14} \\
r_{21} & r_{22} & r_{23} & r_{24} \\
r_{31} & r_{32} & r_{33} & r_{34}
\end{array}\right)\left(\begin{array}{cccc}
\cos \theta & \sin \theta & 0 & 0 \\
-\sin \theta & \cos \theta & 0 & 0 \\
0 & 0 & 1 & 0 \\
0 & 0 & 0 & 1
\end{array}\right)\left(\begin{array}{c}
X_{p} \\
Y_{p} \\
Z_{p} \\
1
\end{array}\right)
$$

Hay que hacer notar que, después de la rotación de las coordenadas 3D iniciales con cada matriz $R$, los puntos con coordenadas $X_{p_{R}}<0$ son filtrados, ya que corresponderían a proyecciones de puntos por detrás de la cámara.
Por último, los valores de coordenadas $\left(\mathrm{X}_{\mathrm{f}}^{\prime} \mathrm{Y}_{\mathrm{f}}^{\prime}\right)$ que se salen de rango de la dimensión de la imagen son también filtrados en el proceso. En nuestro sistema $0<X_{f}^{\prime} \leq 640$ y $0<Y_{f}^{\prime} \leq 512$. Finalmente, un punto $3 \mathrm{D}$ válido tendrá uno o varios valores de temperatura asignados. Esto es debido a que existe un pequeño solapamiento entre imágenes consecutivas por lo que el mismo punto 3D puede estar proyectado en dos imágenes térmicas. En ese caso, el valor final asignado es el valor medio de ambas temperaturas asignadas. Finalmente, se consigue la nube de puntos térmica (NPT).

La Figura 8 muestra la asignación de temperatura para una sola imagen térmica, mientras que la Figura 9 ilustra el resultado de integrar tres imágenes térmicas consecutivas sobre una parte de la nube de puntos.
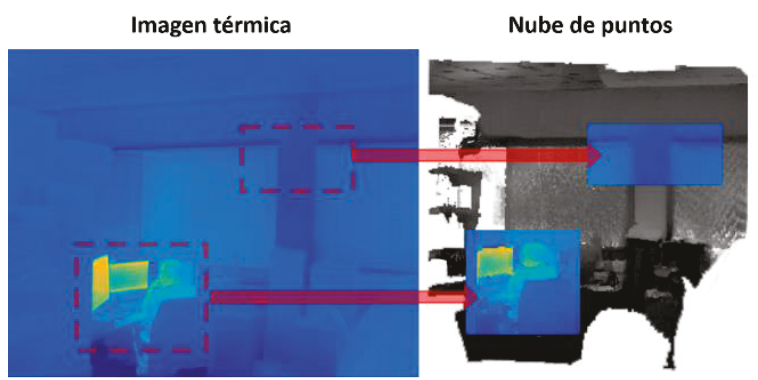

Figura 8: Ilustración de una imagen térmica y asignación de color en la nube de puntos. La figura muestra la asignación de dos partes sobre la nube de puntos.

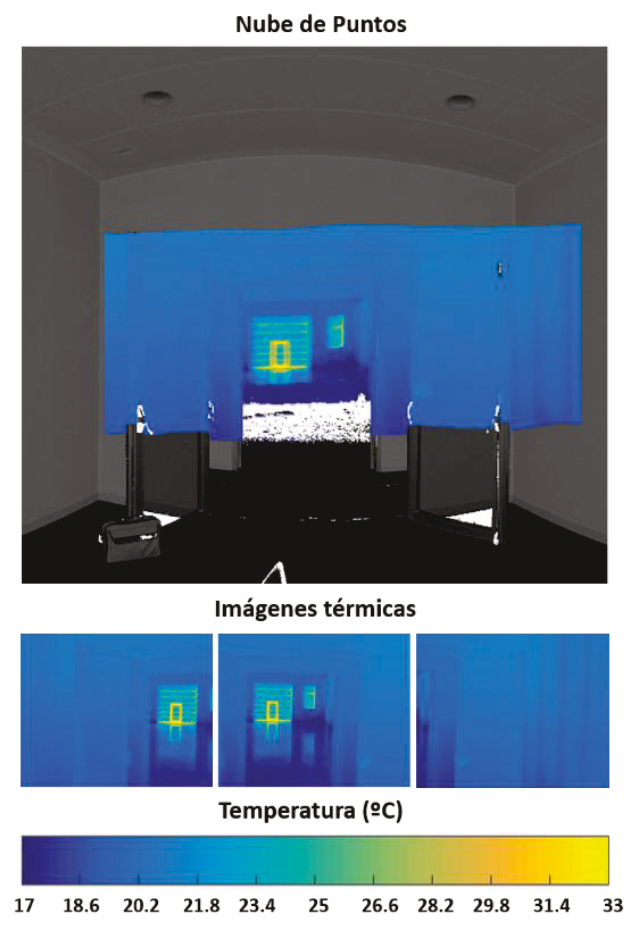

Figura 9. Integración de varias imágenes térmicas en una nube de puntos. 


\subsection{INTEGRACIÓN DE VARIAS NUBES DE PUNTOS TÉRMICAS.}

Como se expuso en la Sección 2, el sistema 3D T-Scan no es capaz de muestrear todo el espacio visible debido a que el rango de $\lambda$ está limitado. Esto causa la existencia de dos conos (inferior y superior) en el que no se registran datos. Por otra parte, debido a oclusiones causadas principalmente por el mobiliario existente, una toma de escáner es insuficiente para generar un modelo 3D térmico completo de la estructura arquitectónica de la escena. Por estas razones, para generar un mapa térmico completo de una escena, se necesitan varias tomas desde varias posiciones.

La integración de varias NPTs tomadas en diferentes posiciones del escáner sigue un procedimiento usual de un registro de nubes de puntos. Se utilizan marcas reflectantes distribuidas en la escena, con el requerimiento de ser visibles desde distintas posiciones.

Para realizar el registro de dos NPTs, se genera una imagen polar de reflectancia y se asocian las marcas correspondientes (Figura 10 a)). Con cuatro o más parejas de marcas, se calcula la matriz de transformación de las NPTs y se pasa a un sistema de referencia común, que normalmente ha sido fijado en la posición de la primera sesión se escaneo.

Este proceso es secuencial desde la primera a la última NPT. En cada iteración se genera una nube acumulada (Figura 10 b)) en la que se llevan a cabo dos procesos.

1. Registro y muestreo con la nube anterior. El muestreo se realiza a través de un filtrado de rejilla de vóxeles (box grid filter), reduciendo el tamaño de la nube acumulada.

2. Obtención de color y temperatura de la nube reducida. Se realiza igualmente recurriendo al promedio de color y temperatura por vóxel.
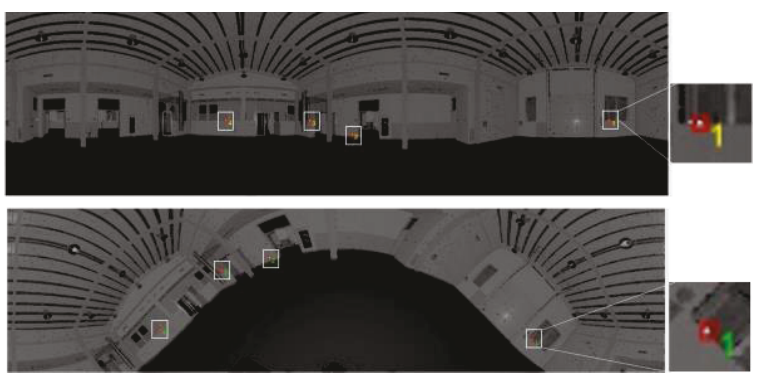

a)

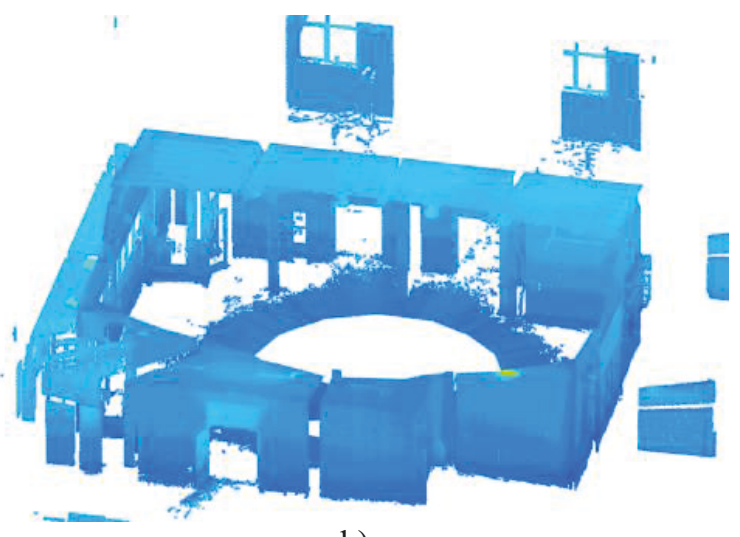

b)

Figura 10. a) Imágenes polares de dos nubes de puntos y dianas reflectantes correspondientes. b) Nube de puntos térmica acumulada reducida.

\section{RESULTADOS EXPERIMENTALES}

El sistema T-Scan ha sido puesto a punto en el 3D Visual Computing \& Robotics Lab de la Universidad de Castilla La Mancha y probado en entornos reales. En este apartado se presentan los resultados obtenidos en un caso de estudio.

Se ha realizado el modelo de nube de puntos térmica de una parte del Instituto de Ciencias de la Edificación en el campus de Cuenca de la Universidad de Castilla La Mancha. Este trabajo se ha realizado en varias sesiones de trabajo durante tres días. En total se realizaron 87 tomas de escáner desde 27 posiciones, con el siguiente volumen aproximado de datos: 435 millones de puntos espaciales de escáner, de los cuáles un $76 \%$ tiene color asignado y un $40 \%$ contiene información térmica. En el proceso de toma de datos se utilizaron 40 dianas reflectantes. La planta y las posiciones del escáner pueden verse en la Figura 11.

El rango en $\boldsymbol{\theta}$ fue de $\left[0,360^{\circ}\right]$, con un paso de barrido del escáner de $0.08^{\circ}$. Se tomaron 15 fotografías para un giro completo del escáner.

Los tiempos invertidos en la toma de datos es el siguiente: toma de datos de escáner (en rango de $360^{\circ}$ ) $47 \mathrm{seg}$, toma de imágenes color y térmicas $60 \mathrm{seg}$.

La Figura 12 muestra el resultado del modelo térmico de una sala del edificio con un pseudocolor insertado. Se aprecian claramente salidas de calefacción en un tono amarillo, así como pequeñas variaciones de color en las paredes. Esta pequeña variación se hace más evidente en la parte baja de la pared donde hay supuestas humedades. 


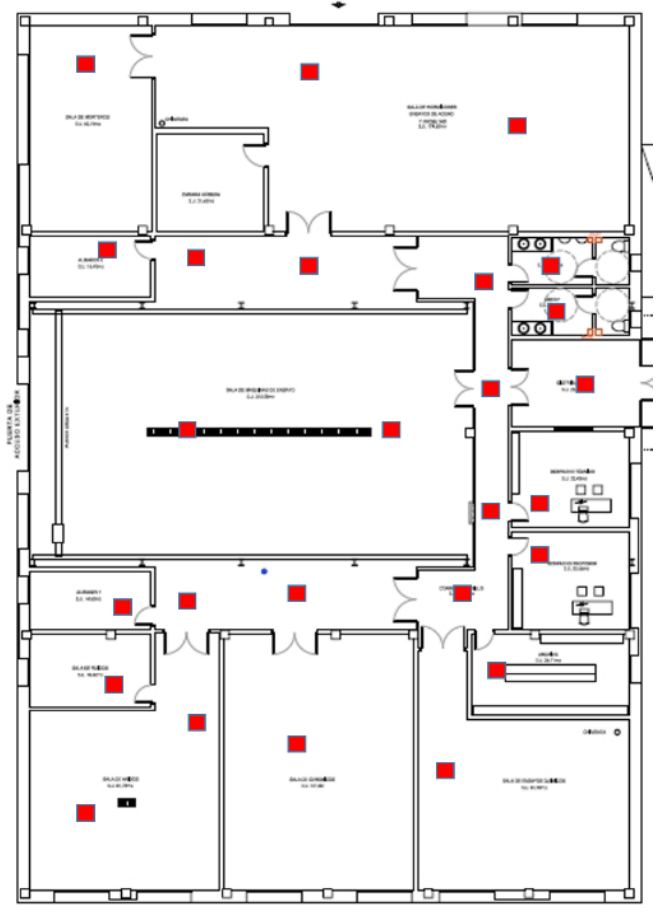

Figura 11. Posiciones del sistema T-Scan en el escenario del trabajo.

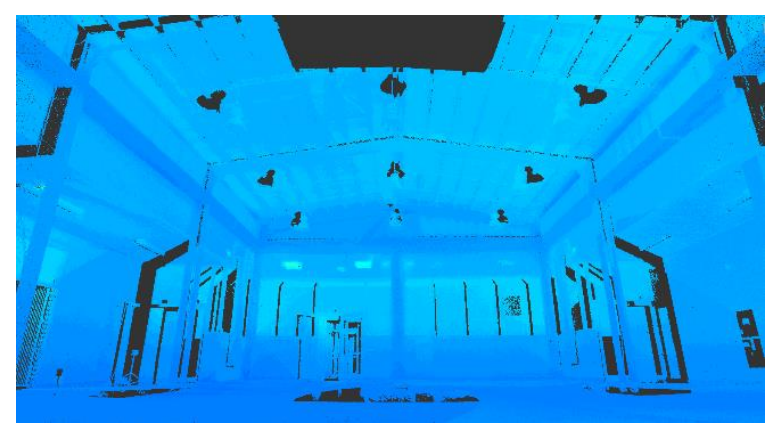

a)

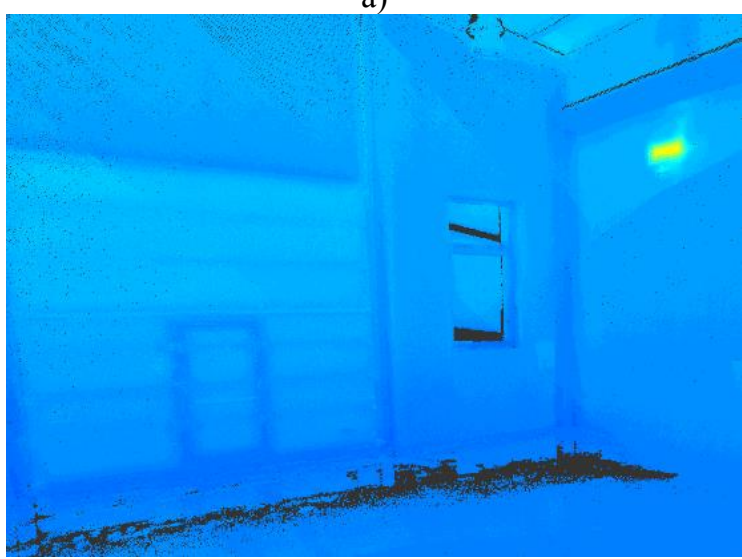

b)

Figura 12. a) Imagen la nube de puntos térmica en una de las salas del edificio. Se aprecia variación de temperatura en distintos componentes del edificio y salidas calientes de aire. b) Detalle de variación de temperatura en la parte inferior de las paredes.

\section{CONCLUSIONES}

El objetivo general del artículo es presentar un sistema sensorial junto con su metodología asociada para obtener modelos de nubes de puntos térmicos de interiores de edificaciones. Este sistema se integra como una primera etapa para generar soluciones de ingeniería inversa sobre la creación automática de modelos 3D BIM térmicos y su monitorización en el tiempo.

El sistema T-Scan se ha diseñado para cubrir el espacio observable mediante integración de varias tomas desde varias posiciones. La nube térmica integrada es muestreada a cada iteración con objeto de no saturar la memoria, obteniendo finalmente un modelo completo asumible para ser posteriormente procesado en la etapa de obtención del modelo semántico térmico del edificio. El sistema ha sido probado en una planta de $40.70 \mathrm{~m} \times 28.45 \mathrm{~m}$ con 18 salas obteniendo resultados muy satisfactorios.

\section{Agradecimientos}

Este trabajo ha sido financiado por los proyectos AEI/FEDER, UE. DPI2013-43344-R (Ministerio Español de Economía y Competitividad), PEII-2014017-P (Gobierno de Castilla la Mancha) y la beca predoctoral UCLM con el número de referencia PREDUCLM16/23.

\section{Referencias}

[1] D. Borrmann, A. Nüchter, M. Đakulović, I. Maurovic, I. Petrović, D. Osmankovic, J. Velagić, A mobile robot based system for fully automated thermal 3D mapping, Adv. Eng. Informatics. (2014).

[2] Y.K. Cho, Y. Ham, M. Golpavar-Fard, 3D as-is building energy modeling and diagnostics: A review of the state-of-the-art, Adv. Eng. Informatics. 29 (2015) 184-195.

[3] D. González-Aguilera, P. Rodriguez-Gonzalvez, J. Armesto, S. Lagüela, Novel approach to 3D thermography and energy efficiency evaluation, Energy Build. 54 (2012) 436-443.

[4] Y. Ham, M. Golparvar-Fard, An automated visionbased method for rapid 3D energy performance modeling of existing buildings using thermal and digital imagery, Adv. Eng. Informatics. 27 (2013) 395-409.

[5] J. Heikkila, O. Silven, A four-step camera calibration procedure with implicit image correction, Proc. IEEE Comput. Soc. Conf. Comput. Vis. Pattern Recognit. (n.d.) 1106-1112.

[6] S. Lagüela, J. Martínez, J. Armesto, P. Arias, Energy efficiency studies through 3D laser scanning and thermographic technologies, Energy 
Build. 43 (2011) 1216-1221.

[7] L. Lopez-Fernandez, S. Lagu ela, D. GonzalezAguilera, H. Lorenzo, Thermographic and mobile indoor mapping for the computation of energy losses in buildings, Indoor Built Environ. 0 (2016) $1-14$.

[8] D. Mader, R. Blaskow, P. Westfeld, C. Weller, Potential of UAV-Based laser scanner and multispectral camera data in building inspection, Int. Arch. Photogramm. Remote Sens. Spat. Inf. Sci. - ISPRS Arch. 2016-Janua (2016) 1135-1142.

[9] J. Rangel, S. Soldan, A. Kroll, 3D Thermal Imaging: Fusion of Thermography and Depth Cameras, in: 2014 Quant. InfraRed Thermogr., 2014.

[10]C. Wang, Y.K. Cho, M. Gai, As-Is 3D Thermal Modeling for Existing Building Envelopes Using a Hybrid LIDAR System, J. Comput. Civ. Eng. 27 (2013) 645-656. 